\title{
Small-strain deformation behaviour of a clay at frozen and unfrozen states: A comparative study
}

\author{
Satoshi Nishimura ${ }^{1, *}$, Shota Okajima ${ }^{2}$, Jinyuan Wang $^{3}$, and Bhakta Raj Joshi ${ }^{2}$ \\ ${ }^{1}$ Hokkaido University, Faculty of Engineering, Japan \\ ${ }^{2}$ Hokkaido University, Graduate School of Engineering, Japan \\ ${ }^{3}$ Wuhan University, State Key Laboratory of Water Resources and Hydropower Engineering Science, China
}

\begin{abstract}
The small-strain deformation behaviour of frozen high-plasticity clay, and the factors influencing it were investigated through parallel tests at frozen and unfrozen states. The first and second series involved temperature-controlled triaxial compression tests on unfrozen and frozen samples, respectively, with accurate strain measurement with local displacement sensors, fully calibrated for cold environment. The small-strain loading was conducted at different axial strain rates and temperatures. At pre-yield small strains in order of $0.001 \%$, Young's modulus was independent of the strain rate, in a same manner as in unfrozen soils. The strain rate only affected the onset of small-scale yielding and the degradation of stiffness after that. The elastic strain range was greater at lower temperature, but the degree of stress-strain non-linearity seen at small strains remained on the whole similar between frozen and unfrozen states. An interesting feature of the frozen clay's stiffness, also confirmed by third test series adopting bender elements, is that it decreases when the soil is frozen from higher effective stress. A simple model was proposed to explain this feature.
\end{abstract}

\section{Introduction}

Artificial ground freezing technique is an effective method in securing sufficient ground stability or preventing ground water seepage during underground construction. Prediction of the ground behaviour during/after freezing is attempted by increasingly sophisticated thermo-hydro-mechanical coupled models in recent years [1]. Whilst the mechanical properties of frozen soils have been widely studied over many years [2], the laboratory testing practice is yet to meet demands of the complex coupled models. Critical Statebased constitutive models of frozen soils $[1,3,4]$ require carefully designed laboratory tests in which the state (as defined by the stress and density) and its history are clear for their calibration and further development $[5,6]$. Reappraising the frozen soil behaviour in this new perspective, it is evident that more fundamental study on frozen soil stiffness is necessary. Although the literature is informative on the general effects of temperature, strain rate and post-freezing confining stress [7,8], the relatively large strains at which the secant stiffness is defined prevents understanding the elastic and plastic components of the strains separately, and hence the nature of the measured stiffness. Although the above early studies were followed, for example, by advanced work on sand by an MIT team [9-11], much is still unknown of the frozen soils' small-strain stiffness. Understanding and modelling stiffness in a general manner under intransient temperature, strain rate and stress-density states is important not only in predicting the ground movement, but also in assessing the distress to adjacent structures. This is because the artificial ground freezing is mostly conducted in relatively deep ground where confined kinematics prevails and hence the stress regime is sensitive to the soil stiffness. A frozen soil model incorporating an appropriate submodel for state-dependent stiffness will be capable of addressing the above problem along with the stability (strength) problem.

This study reports a laboratory study on stiffness of a frozen high-plasticity clay through both static and dynamic measurements. A clay was adopted as test material mainly because the stress-density state is more clearly defined - the Normal Compression Line; NCL, the Critical State Line; CSL, Over-Consolidation Ratio; OCR, are all well-defined as compared to sands and intermediate soils. This feature of clay will assist interpreting and modelling the state-dependent stiffness. The static measurement was performed on frozen samples in a temperature-controlled triaxial apparatus with semi-local instrumentation for axial strain and extremely precise axial loading device, enabling the strain to be resolved to order of $0.001 \%$. The dynamic measurement involved shear wave velocity identification with bender elements in a separate, purpose-designed apparatus but under same conditions as the static tests. This study, uniquely, conducted another series of tests on unfrozen samples in parallel with the frozen test series. By relating the observed stiffness to the state, a simple

\footnotetext{
Corresponding author: nishimura@eng.hokudai.ac.jp
} 
conceptual model to interpret the effect of pre-freezing effective stress on the initial small-strain stiffness is proposed.

\section{Tested material and apparatus}

\subsection{Tested material and sample preparation}

Kasaoka clay, a high-plasticity clay commercially available in a powder form, was adopted for the tests. The plastic and liquid limits are $22.5 \%$ and $59.6 \%$, respectively. The clay is $60 \%$ smaller than $0.002 \mathrm{~mm}$, and $100 \%$ smaller than $0.075 \mathrm{~mm}$ by weight. It was reconstituted from a slurry state with the water content, $w$, of $100 \%$ in one-dimensionally pre-consolidation. After being removed from the consolidometer, the clay was trimmed to $30 \mathrm{~mm}$ in diameter and $60 \mathrm{~mm}$ in height for frozen tests, and $50 \mathrm{~mm}$ in diameter and $100 \mathrm{~mm}$ in height for unfrozen tests. The specimens were then transferred to the testing apparatus. More information on the clay, such as mineralogy, is provided in [5].

\subsection{Triaxial apparatus for frozen and unfrozen samples}

Two different triaxial machines were used, one for frozen conditions and the other for unfrozen conditions. The two machines are described in [5] and [12], respectively. Common to both are that their axial rams are driven by extremely precise direct-drive motors with resolution of $10^{-5} \mathrm{~mm}$, and that they are equipped with highly precise full- or semi-local axial strain transducers. While the 'unfrozen' triaxial system had a pair of LVDTs for fully local axial strain measurements, the 'frozen' triaxial system adopted a pair of proximity transducers sensing on-specimen metal targets but itself anchored to the cell base, as illustrated in Fig. 1. The
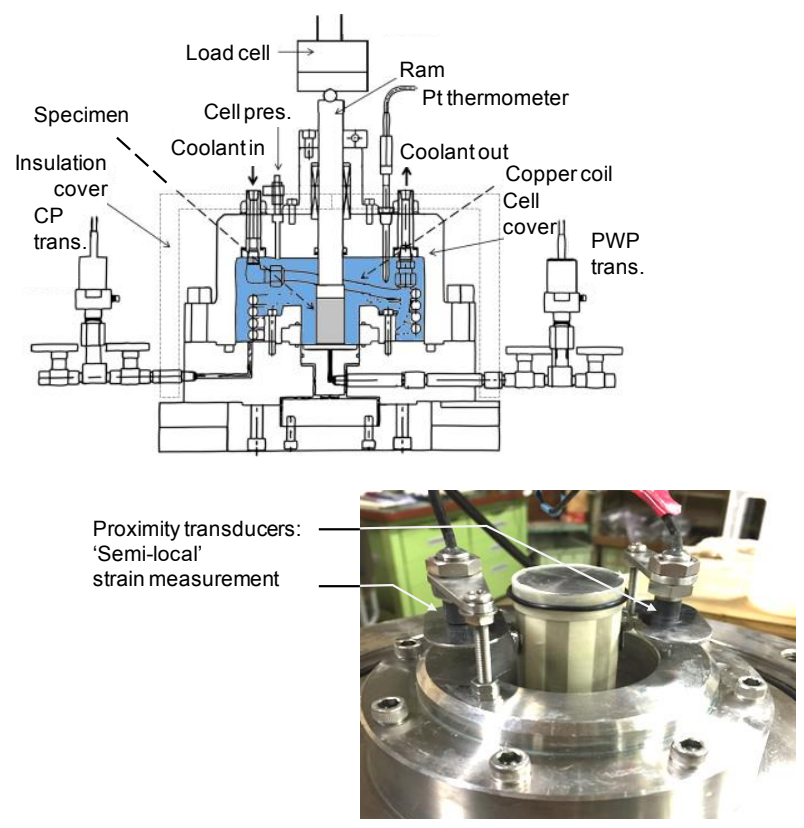

Fig. 1. Triaxial apparatus for frozen soil testing, with a pair of proximity transducers functioning in the refrigerant.

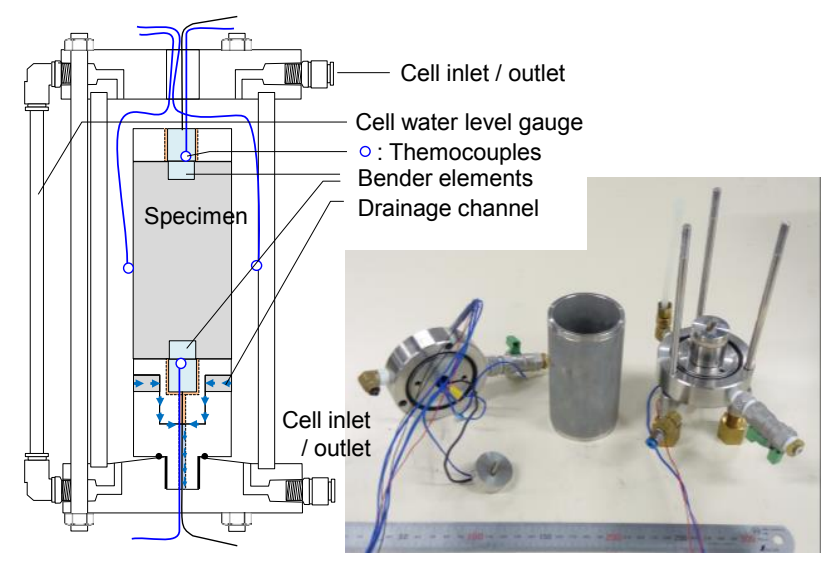

Fig. 2. Miniature freezing cell for bender element testing allowing freezing after isotropic consolidation.

latter is hence only 'semi-' local - however, the specimen-pedestal bedding error under frozen conditions is considered to be minor compared to machine component compliances, which were avoided in this arrangement.

\subsection{Miniature freezing cell for bender element testing}

A miniature freezing cell was newly developed for bender element probing of shear wave velocity in the frozen samples under isotropic pressure. The cell had an inner diameter of $53 \mathrm{~mm}$, housing a $30 \mathrm{~mm}$-diameter and $60 \mathrm{~mm}$-height specimen, as illustrated in Fig. 2. This cell allows isotropic consolidation of the specimen in a same manner as in triaxial apparatus. The cell water could then be drained slowly, keeping the specimen pressurised by incoming compressed air. The cell is sufficiently small to be directly immersed in a coolant bath, typically prepared at $-25^{\circ} \mathrm{C}$. The air inside is then promptly cooled, and the freezing front reaches the specimen core within an hour. The bender elements were installed to measure the shear wave velocity travelling in the axial direction.

\section{Testing scheme and procedures}

\subsection{Unfrozen triaxial test series: Series U}

For interpreting the state-dependency of the frozen Kasaoka clay's small-strain stiffness, formulation of the stiffness at unfrozen states was sought as reference. Series U, conducted on unfrozen samples, involved three specimens isotropically consolidated to different levels of effective stress; $p^{\prime}=100,200$ and $400 \mathrm{kPa}$ each (test cases U100, U200 and U400, respectively). They were allowed to isotropically swell after reaching the above stresses, and the stiffness was measured along these paths by static undrained cyclic loading with small axial strain amplitudes $(0.001 \%$ and $0.03 \%)$ at constant axial strain rates of $0.001,0.01$ and $0.1 \% / \mathrm{min}$. The NCL and the swelling lines from Series $U$, as well as the stiffness probe points, are illustrated in Fig. 3. The NCL was slightly different from that observed in another next test series (Series F), possibly due to the larger specimen size. 


\subsection{Frozen triaxial test series: Series $F$}

The frozen test series, named Series F, followed a scheme similar to Series $\mathrm{U}$, involving three specimens isotropically consolidated to $p^{\prime}=100,200$ and $400 \mathrm{kPa}$ each (test cases F100, F200 and F400, respectively). The specimen was then quickly frozen to $-15^{\circ} \mathrm{C}$ by replacing the cell water with pre-cooled refrigerant. The quick freezing kept the inhomogenisation of the specimen due to ice-soil segregation to minimum and results in largely uniform water content across the specimen [5]. The process was undrained, and the specimen volume was expected to increase as a large proportion of pore water turned ice. After reaching equilibrium at $-10^{\circ} \mathrm{C}$, undrained small-strain compression with externally measured axial strain of $0.25 \%$ at $0.001,0.01$ and $0.1 \% /$ min was performed. Note that the ram was not rigidly connected to the loading platen in this 'frozen' triaxial cell, and the loading was in compression only (i.e. single-sided cyclic loading). This process was repeated at -5 and $-2^{\circ} \mathrm{C}$ for the same specimen. As the load cell was placed outside the cell, the axial stress measurement was affected by the ram-bush friction. This effect turned out to be unexpectedly large, despite the significant stiffness of the specimen and hence the relatively large compression force involved in the measurement. This problem was eventually avoided by lifting the ram until it detached from the platen, and then lowering it again at a constant rate, thus measuring the friction-induced offset before it met the platen. Without such care, the stiffness would be overestimated by a factor of 2-3.

\subsection{Frozen bender element test series: Series B}

The bender element test series on the frozen samples, named Series B, was conducted in the miniature cell introduced earlier, following a scheme similar to Series F. Three specimens, each of which was consolidated to either $p^{\prime}=100,200$ or $400 \mathrm{kPa}$, were tested. The shear wave velocity was firstly measured at $-10^{\circ} \mathrm{C}$, and then at -5 and $-2^{\circ} \mathrm{C}$, following the scheme in Series F. The frequency range appropriate for the bender element excitation was $50-80 \mathrm{kHz}$. The measured shear wave velocity, $V_{s}$, was converted into the shear modulus, $G$, via $G=\rho V_{s}^{2}$, and then to Young's modulus, $E$, via $E=3 G$.

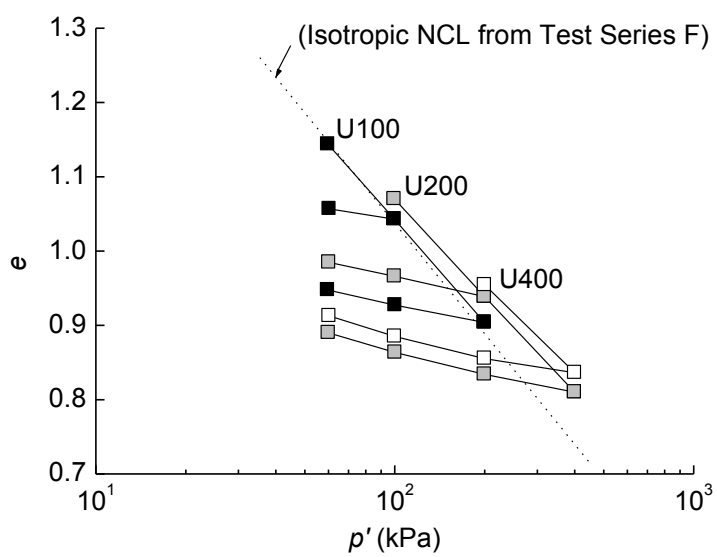

Fig. 3. NCL and swelling lines for Series U (unfrozen series) and stiffness measurement points.

\section{Test results and observations}

\subsection{Small-strain stiffness of unfrozen clay}

Small-strain deformation characteristics of unfrozen clays have been widely studied and do not require detailed discussion. Series U is helpful, however, for two purposes; (i) To compare and observe a parallel with the stiffness characteristics at frozen states, and (ii) parameterising the state-dependency of the unfrozen stiffness as ingredient of frozen soils' stiffness model. Fig. 4 shows typical results from $\mathrm{U} 400$ at $\mathrm{OCR}=6.7$. Consistent with, for example, [13], the initial Young's modulus, $E_{0 u f}$ (the subscript $u f$ referring to unfrozen) was independent of the strain rate and only the onset of stress-strain non-linearity was affected by the rate. From all the three tests in Series U, the $E_{0 u f}$ values measured on the NCL are plotted against $p^{\prime}$, both normalised by a reference pressure $p_{r}=1 \mathrm{kPa}$, in Fig. 5(a). In Fig. 5(b), the $E_{0 u f}$ values measured at over- consolidated states are

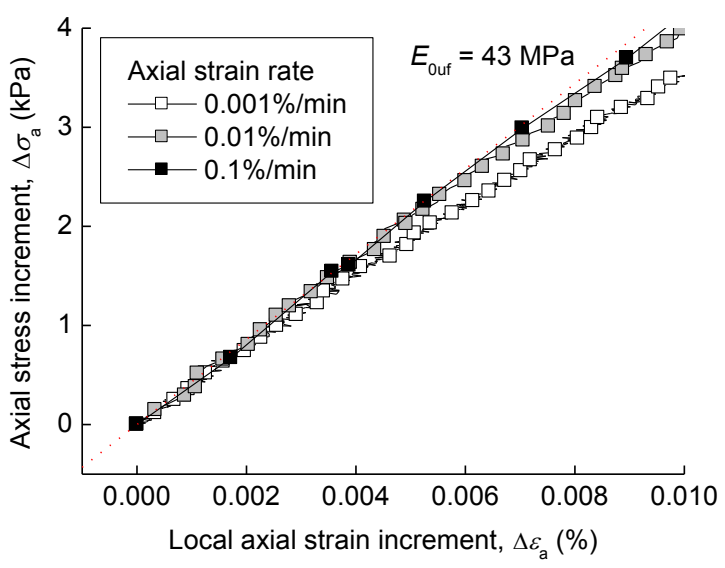

Fig. 4. Typical axial stress - strain relationship at small strains for unfrozen Kasaoka clay (example at $p^{\prime}=60 \mathrm{kPa}, \mathrm{OCR}=6.7$ ).


Fig. 5. Initial small-strain Young's modulus (normalised) in unfrozen Kasaoka clay; (a) at OCR=1, (b) at various OCR . 
normalised by the values on the NCL, $E_{0 u f N C}$. In both plots, the power law curves fit the data points well, leading to the following expression;

$$
E_{0 \mathrm{u} f N C}=\operatorname{Ap}_{r}\left(p^{\prime} / p_{r}\right)^{a} \mathrm{OCR}^{b}
$$

Where $A, a$ and $b$ are constants, as determined in Fig. 5 for Kasaoka clay. This expression was originally proposed in [14] for $G$.

\subsection{Small-strain stiffness of frozen clay}

The axial stress-strain relationships observed for frozen Kasaoka clay samples in Series F are shown in Fig. 6 for three temperatures and three loading rates. Only the data from Test F400 (i.e. frozen from $p^{\prime}=400 \mathrm{kPa}$ ) are shown here. For each temperature, the initial small-strain Young's modulus, $E_{0}$, is independent of the strain rate and uniquely determined. However, the strain rate, along
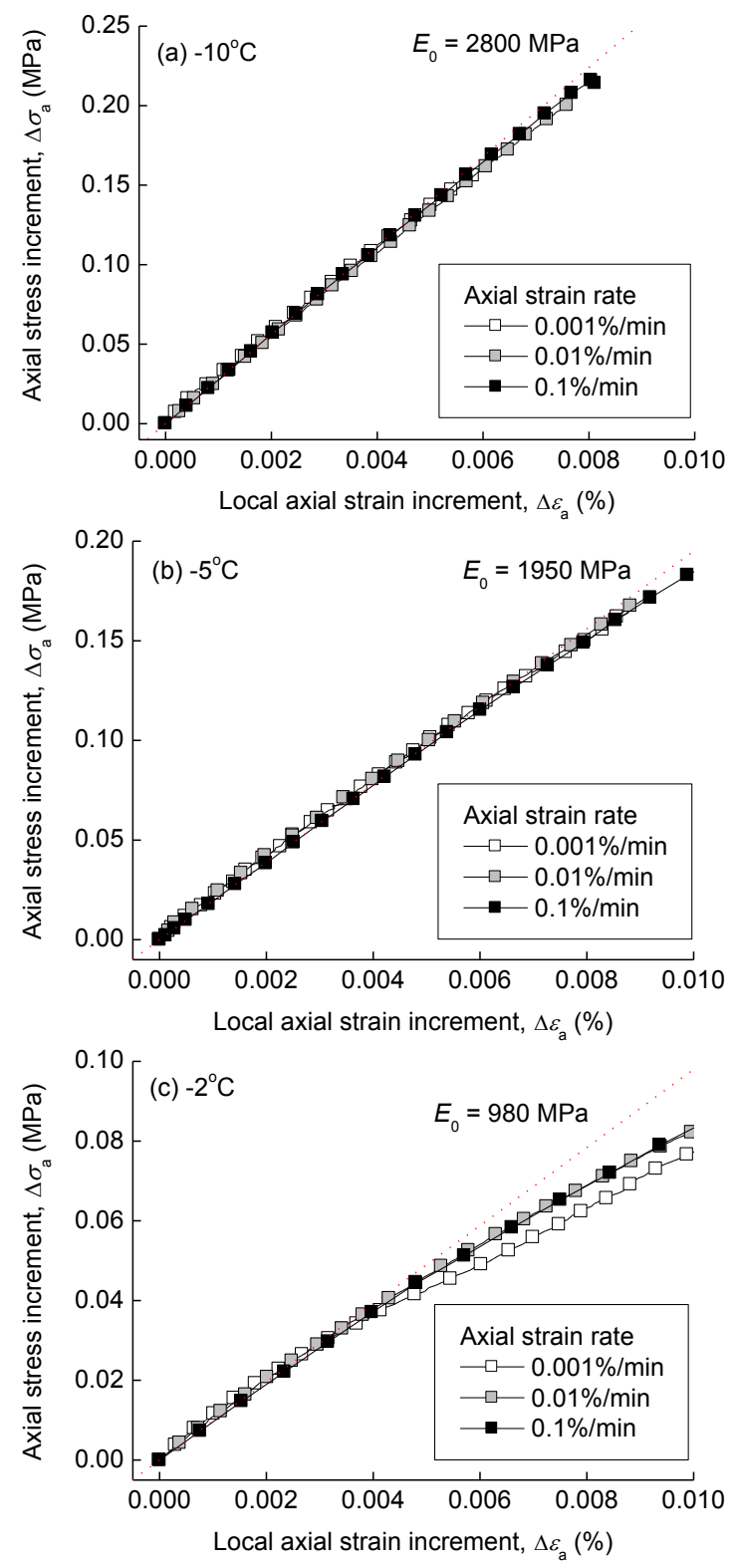

Fig. 6. Axial stress - strain relationships for Test $\mathrm{F} 400$ (frozen from $p^{\prime}=400 \mathrm{kPa}$ ); (a) at $-10^{\circ} \mathrm{C}$, (b) at $-5^{\circ} \mathrm{C}$, (c) at $-2^{\circ} \mathrm{C}$.
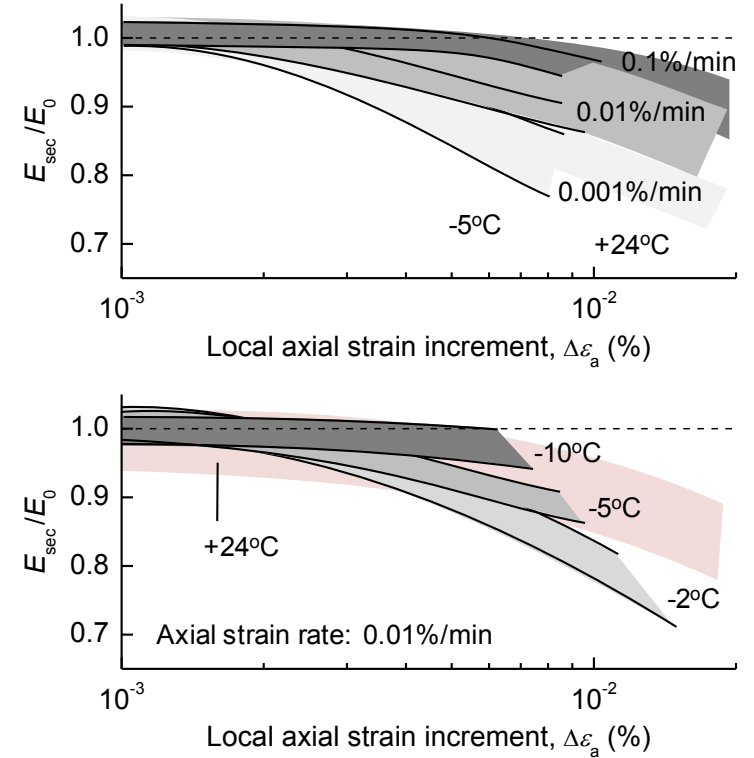

Fig. 7. Degradation of secant Young's modulus against strain, each shown as envelope of all the three tests from Series U or F: Strain rate effect (top) and temperature effect (bottom).

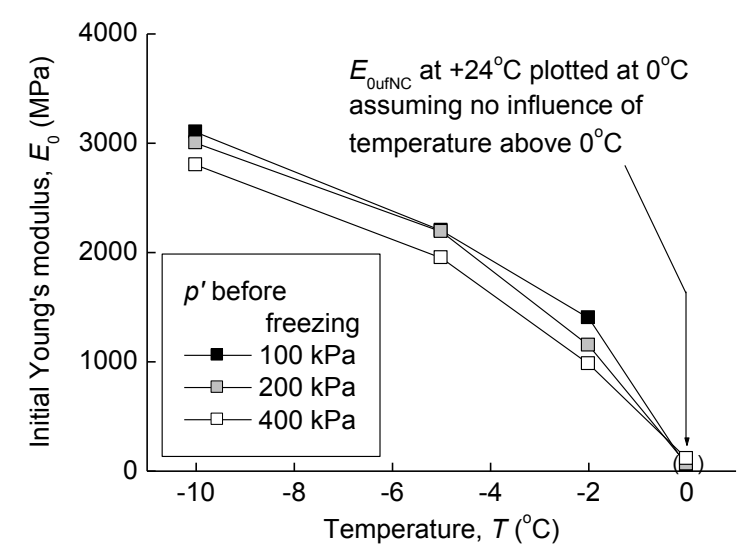

Fig. 8. Variation of statically measured initial Young's modulus for frozen and unfrozen Kasaoka clay with temperature and pre-freezing $p^{\prime}$ (Test Series U and F).



Fig. 9. Variation of shear wave velocity-based initial Young's modulus for frozen and unfrozen Kasaoka clay with temperature and pre-freezing $p^{\prime}$ (Test Series B). 
with temperature, clearly affects the strain level at which stress-strain non-linearity appears. The higher the strain rate is, the greater strain is required for the onset of the non-linearity. These features are essentially same as those observed for the unfrozen states. However, the degree of the strain rate effect varies significantly depending on the temperature. The effect, or viscosity, increases markedly at warmer temperature $\left(-2^{\circ} \mathrm{C}\right)$, while at $-10^{\circ} \mathrm{C}$ it is even less significant than unfrozen states (Fig. 4). These characteristics were common to the specimens frozen from $p^{\prime}=100$ and $200 \mathrm{kPa}$ too, as seen in the normalised secant modulus $E_{\text {sec }} / E_{0}$ shown in Fig. 7 as envelopes of the three tests from Series U or Series F (for clearer presentation, the figure is divide into two, showing the strain rate effect and the temperature effect in the top and bottom plots, respectively). Ice is commonly considered as highly viscous material with a strong tendency to creep. However, the above results show that ice-soil mixture (i.e. frozen soil) is appropriately described by time-independent elasticity for strain smaller than $\sim 0.001-0.002 \%$ below $-2^{\circ} \mathrm{C}$. Its small-strain deformation behaviour is qualitatively same as that of unfrozen states, if not quantitatively.

The values of $E_{0}$ obtained from the static loading is plotted against the temperature and the pre-freezing $p^{\prime}$ value in Fig. 8 ( $E_{0}$ was assumed to be constant above $0^{\circ} \mathrm{C}$ ). In addition to the obvious influence of temperature, it is noteworthy that the pre-freezing $p^{\prime}$ affects the stiffness in a negative manner. A same trend is observed in the $E_{0}$ values evaluated from shear wave velocity measured by bender elements (Series B), as shown in Fig. 9. The two types of measurement are compatible, both qualitatively and quantitatively. Although the pressuremelting is often invoked to explain the mechanical degradation of frozen soils at higher pressure, this mechanism should be ruled out here, since the prefreezing back pressure was same at $200 \mathrm{kPa}$ in all the tests. The implication is that more consolidated (i.e. higher $p^{\prime}$ ) and hence denser clay is softer when frozen. A simple model is considered in the next section to make sense of this apparently counter-intuitive result.

\section{Interpretation of frozen soil stiffness based on ice and unfrozen soil stiffness}

Frozen soil, even if saturated with water, is a complex mixture of multiple phases and materials, with frozen and unfrozen water occupying the pores at proportions that vary with temperature and pressure. Although a theoretical framework that captures such a complex system is yet unavailable, a simple model explains the observed influence of pre-freezing $p^{\prime}$. Here we assume a parallel system of soil skeleton and pore ice resisting the shear deformation, thus decomposing the Young's modulus (equivalent to shear modulus under undrained conditions, as in this study);

$$
E_{0}=E_{0 u f}+E_{p i}
$$

where $E_{p i}$ represents Young's modulus due to the pore ice, and it is assumed to be proportional to its volumetric content;

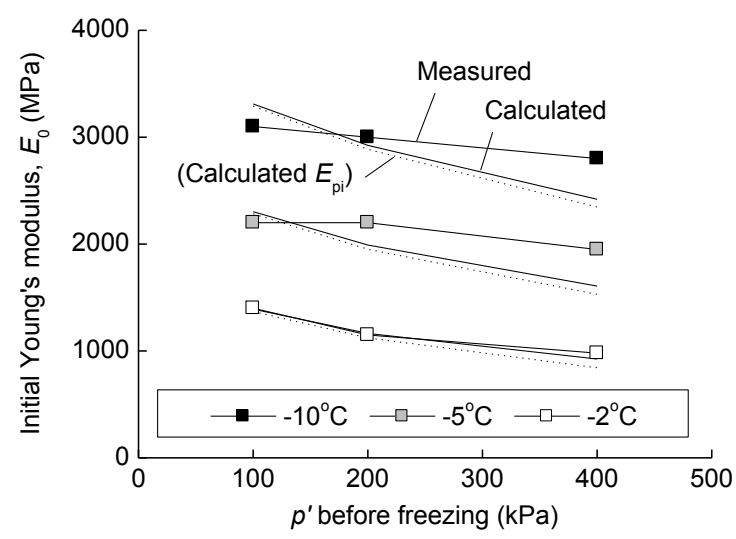

Fig. 10. Observed and modelled variation of initial Young's modulus with pre-freezing $p^{\prime}$.

$$
E_{p i}=n S_{i} E_{\text {ice }}
$$

where $n$ is the porosity, $S_{i}$ is the degree of ice saturation (volume of ice / volume of pore) and $E_{i c e}$ is Young's modulus of ice. The $n$ and $S_{i}$ after freezing could be calculated from the unfrozen water content-temperature relationship separately obtained from Nuclear Magnetic Resonance (NMR) probes [5] and by accounting for the $9 \%$ volume increase due to the phase change (liquid to ice) of water. The $E_{\text {ice }}$ value was assumed to be 8000 , 6000 and $4000 \mathrm{MPa}$ for $-10,-5$ and $-2^{\circ} \mathrm{C}$, respectively. The soil skeletal stiffness, or the stiffness of the unfrozen soil, $E_{0 u f}$, was evaluated by using Equation (1) and assuming that the effective stress is related to the void ratio $e$ just in a same manner as in unfrozen states (i.e. the NCL and the unloading curve common to frozen and unfrozen states; see [6] for more detail). The computed values of $E_{0}$ are compared with those observed from the experiments. Although the model tends to slightly overestimate the pre-freezing $p^{\prime}$ effects, it explains the trend generally well. Also shown in the same figure is the calculated $E_{p i}$. The close values of $E_{p i}$ to $E_{0}$ means that, in this model, the stiffness predominantly derives from that of the pore ice. The pre-freezing consolidation leads to less volumetric proportion of the pore ice, and hence the reduced overall stiffness. Increasing prefreezing $p^{\prime}$ leads to higher soil skeletal stiffness $E_{0 u f}$ according to Equation (1) but its effect is relatively small in the soil-ice mixture.

\section{Summary}

Small-strain deformation characteristics of frozen highplasticity clay were presented as measured both in static and dynamic manners. A highly accurate loading system and local instrumentation were adopted for static loading, while a newly designed miniature freezing cell was adopted for bender element testing on frozen specimens. Three different loading rates and three different temperatures were set in the static loading tests for each of specimens frozen from the isotropic effective stress of $p^{\prime}=100,200$ or $400 \mathrm{kPa}$. A similar series was run on unfrozen specimens so that the frozen soil behaviour could be characterised with the unfrozen behaviour as 
reference. To summarise the main outcomes of this study:

1. The initial Young's modulus for unfrozen clay samples under isotropic loading/unloading could be well interpreted with a conventional framework expressing it as function of soil state (i.e. the effective stress and overconsolidation ratio).

2. By adopting high-precision proximity transducers that functions in subzero-temperature refrigerant, the static stress-strain behaviour of frozen clay was characterised below $0.01 \%$ axial strain. The small-strain deformation characteristics were essentially similar to those observed for unfrozen samples; the initial Young's modulus was independent of the strain rate, while the effects appear after the onset of stress-strain non-linearity at strains larger than $0.003 \%$. The higher temperature $\left(-2^{\circ} \mathrm{C}\right.$, compared to $-5^{\circ} \mathrm{C}$ ) accentuated the strain rate effect after this strain level.

3. The Young's modulus values evaluated for frozen samples based on shear wave velocity were compatible with the statically measured values.

4. Both statically and dynamically measured stiffness showed a tendency to decrease as the pre-freezing effective stress increased, meaning that wellconsolidated clay has lower stiffness when being frozen, despite its higher density. This counter-intuitive phenomenon was explained by a mechanism of reduced amount of pore ice having a dominant effect over increased soil skeletal stiffness.

This study was conducted with JSPS KAKENHI Grant-in-Aid (15K14025).

\section{References}

1. F. Casini, A. Gens, S. Olivella, G.M. Viggiani, Env. Geotech. 3, 3 141-154 (2014)

2. O.B. Andersland, B. Ladanyi, Frozen ground engineering, John Wiley \& Sons. (2004)

3. S. Nishimura, A. Gens, S. Olivella, R.J. Jardine, Géotechnique 59, 3 159-171 (2009)

4. S.A. Ghoreishian Amiri, G. Grimstad, M. Kadivar, S. Nordal, Can. Geotech. J. 53, 10 1646-1657 (2016)

5. J. Wang, S. Nishimura, T. Tokoro, Soils and Found. 57, 2 194-210 (2017)

6. S. Nishimura, J. Wang, Géotechnique, ahead of print, DOI: 10.1680/jgeot.17.P.104 (2018)

7. Y. Zhu, D.L. Carbee, Cold Reg. Sci. Tech. 9, 1 3-15 (1984)

8. V.R. Parameswaran, Can. Geotech. J. 17, 1 74-88 (1980)

9. G.R. Andersen, C.W. Swan, C.C. Ladd, J.T. Germaine, Can. Geotech. J. 32, 3 428-451 (1995)

10. G. Da Re, Ph.D. thesis, Massachusetts Institute of Technology (2000)

11. C. Swan, Sc.D. thesis, Massachusetts Institute of Technology (1994)
12. S. Nishimura, Soils and Found. 54, 3 364-376 (2014)

13. F. Tatsuoka, R.J. Jardine, D. Lo Presti, H. Di Benedetto, T. Kodaka, 14th Int. Conf. Soil Mech. Found. Eng., Hamburg, 2129-2164 (1999)

14. G. Viggiani, J.H. Atkinson, Géotechnique 45, 2 249265 (1995) 